NASA/TM-2001-210949

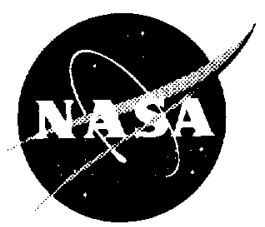

\title{
Mathematical Analysis of Space Radiator Segmenting for Increased Reliability and Reduced Mass
}

\author{
Albert J. Juhasz \\ Glenn Research Center, Cleveland, Ohio
}

Prepared for the

31st International Conference on Environmental Systems sponsored by the Society of Automotive Engineers

Orlando, Florida, July 9-12, 2001

National Aeronautics and

Space Administration

Glenn Research Center 
Available from

NASA Center for Aerospace Information 7121 Standard Drive

Hanover, MD 21076
National Technical Information Service 5285 Port Royal Road Springfield, VA 22100

Available electronically at http://gltrs.grc.nasa.gov/GLTRS 


\title{
Mathematical Analysis of Space Radiator Segmenting for Increased Reliability and Reduced Mass
}

\author{
Albert J. Juhasz \\ National Aeronautics and Space Administration \\ Glenn Research Center \\ Cleveland. Ohio 44135
}

\begin{abstract}
Spacecraft for long duration deep space missions will need to be designed to survive micrometeoroid bombardment of their surfaces some of which may actually be punctured. To avoid loss of the entire mission the damage due to such punctures must be limited to small, localized areas. This is especially true for power system radiators, which necessarily feature large surface areas to reject heat at relatively low temperature to the space environment by thermal radiation. It may be intuitively obvious, that if a space radiator is composed of a large number of independently operating segments, such as heat pipes, a random micrometeoroid puncture will result only in the loss of the punctured segment, and not the entire radiator. Due to the redundancy achieved by independently operating segments, the wall thickness and consequently the weight of such segments can be drastically reduced. Probability theory is used to estimate the magnitude of such weight reductions as the number of segments is increased. An analysis of relevant parameter values required for minimum mass segmented radiators is also included.
\end{abstract}

\section{INTRODUCTION}

The literature on space radiator survivability (e.g., English and Guentert 1961; Juhasz 1998) confirms logical reasoning by indicating that if a space radiator were composed of a multiplicity of independently operating segments, a random micrometeoroid puncture of the radiator would result in the loss of only the punctured segment, not the entire radiator. Hence, the strategic advantage of segmented radiators for space service applications is obvious. Such parallel redundancy can be achieved with pumped loop heat rejection radiators dividing a single closed circuit carrying cooling fluid into a number of independent parallel circuits. But the need for separate circulating pumps, valves, and fluid ducts makes such a system unwieldy for more than a few redundant loops. A much more efficient approach is to utilize the inherent parallel redundancy that heat pipes offer for space heat rejection applications. Since each heat pipe with its extended heat transfer surface fins can be considered as a radiator element that is independent of all other elements and thus expendable the advantage of a heat pipe radiator in terms of modularity and minimum complexity is apparent. Since the design of a space radiator can accommodate the loss in functionality of a certain fraction of elements over the life of a given mission, much thinner walls can be specified. As a result dramatic savings in weight can be realized when compared to a nonsegmented radiator having the same overall survival probability.

Hence there is a clear strategic advantage of segmenting large surface area radiators for space applications. The purpose of this paper is to use basic probability theory in examining the tradeoff between number of segments and the segment wall thickness in order to achieve minimum radiator mass, subject to certain assumptions regarding the total number of segments $N_{s}$ that are required to survive till completion of a mission. An analysis of requirements for minimum mass systems is also included in the appendix to resolve some initial discrepancies with results reported in the literature.

\section{MATHEMATICAL ANALYSIS}

The tradeoff between the number of segments and the segment wall thickness, in order to achieve minimum radiator weight, can be derived on the basis of a simple probability analysis:

If we assume that for each of $N$ identical radiator segments the probability of not being punctured is $p$, then the probability $S$ of having at least $N_{s}$ segments not punctured during the spacecraft radiator's design life is given by the cumulative binomial distribution as

$$
S=\sum_{n=N s}^{n=N} \frac{N !}{n !(N-n) !}(1-p)^{N-n} p^{n}
$$

For each segment the probability $p$ of no puncture is given by

$$
p=e^{-h a t}, \text { or } \ln (1 / p)=h a t ; \text { and } h=\left[\frac{\ln (1 / p)}{a t}\right]
$$

where $h$ is the average puncture rate per unit time and unit area; $a$ is the vulnerable area of each segment; and $t$ 
is the required useful life of the radiator. Note, that for large numbers of segments $N$, the value of $p \sim N_{s} / N$.

The puncture rate $h$ is a function of both the depth of penetration and the rate of flux of meteoroids. Summers and Charters (1959) observed that the wall thickness, $T_{w}$, which a meteoroid will barely penetrate, is proportional to the cube root of the meteoroid mass, $m$. Moreover, Whipple (1958) showed that the meteoroid flux (number frequency) is inversely proportional to meteoroid size (i.e., mass). Assuming that the meteoroid velocity is essentially independent of mass, we can express these trends as a simple relationship:

$$
h \sim m^{-1} \sim T_{w}^{-3} ; \text { or } T_{w} \approx h^{-1 / 3}
$$

Using (2) the wall thickness can be written as

$$
T_{w} \approx\left[\frac{\ln (1 / p)}{a t}\right]^{-1 / 3}
$$

Multiplying the numerator and denominator of (3.1) by $\left(N_{s}\right)^{-1 / 3}$ permits rewriting the approximation as

$$
T_{w} \approx\left[N_{s} \ln (1 / p)\right]^{-1 / 3}\left[N_{s} a t\right]^{1 / 3}
$$

With the objective of minimizing the initial weight of the vulnerable radiator surface material while ensuring a specified nonpunctured area at the end of radiator design life, we can write the following expression:

$$
W / N_{s} a=\rho T_{w} N / N_{s}
$$

Equation (4) can be recast as

$$
W=\left(N_{s} a\right) \rho\left(N / N_{s}\right) T_{w}
$$

where $W$ is the initial weight, $\rho$ is the density of the radiator surface material, and $N_{s} a$ is the required final area. In minimizing $W /\left(N_{s}\right.$ a), for simplicity, only the weight of the vulnerable surface area is considered. The fin area, manifold ducting, and structure are not taken into account. However, since the mass ratio of such peripheral components to vulnerable radiator mass can be readily determined, one can apply the proper correction factor to arrive at an overall radiator mass. Also, since material density, $\rho$, may be assumed to be a constant for a given radiator, it becomes an invariant in the analysis. Using (4) and (3.2) a relative weight $w$ can then be defined as

$$
w \equiv\left(N / N_{s}\right)\left[N_{s} \ln (1 / p)\right]^{-1 / 3}
$$

Then by substituting (5) and (3.2) into (4.1) the expression for total weight shown can be approximated as

$$
W \sim\left(N_{s} a\right) w\left(N_{s} a t\right)^{1 / 3}
$$

Since the required radiator area at the end of the mission and the required mission life are input parameters for the analysis and thus are known a priori, the product $\left(N_{s} a t\right)$ can be considered a constant. Segmenting influences the weight per unit area $\left(W / N_{s}\right.$ a) required at the end of life by affecting the relative weight term $w$. The term $w$, that is relative weight per unit segment area, decreases continually as the number of segments increases.

A brief paper on radiator segmenting by English and Guentert (1961) reported, without showing assumptions or calculation details, that for large values of $N$ the minimum weight per unit segment area will occur at

$$
\left(N_{s} / N\right)_{\min }=e^{-1 / 4}=0.7788
$$

This result differed somewhat with the detailed analysis performed by the author (Juhasz 1998) under the constraint that a constant number of surviving segments $N_{s}$ was required. In this case, the value of $N_{s} / N$ for minimum weight per unit segment area was found to be

$$
\left(N_{s} / N\right)_{\min }=e^{-1 / 3}=0.7165
$$

To resolve the discrepancy the minimum relative mass calculations are performed here in detail for the alternative constraint of a constant number of initial segments $N$. Two alternate approaches for each of the two constraint assumptions are included in the Appendix of this report. As shown in section B of the appendix, holding the initial number of segments $N$ constant, will indeed result in minimum $w$ at $N_{s} / N=$ $e^{-1 / 4} \equiv 0.7788$.

Both assumptions, constant $N_{s}$ or constant $N$ are valid. But the case that specifies a required number of surviving segments $N_{s}$ is somewhat more conservative, since the number of initial segments for a minimum mass system would have to be about 8 percent. However, the relative mass versus segment ratio curves are fairly flat near their minimum values, and both assumptions yield results which are approximately within $a \pm 4$ percent band of 0.75 . Hence, for design guidelines, the value of $N s / N$ for minimum weight per unit segment area can be assumed as

$$
\left(N_{s} / N\right)_{\min } \sim 0.75
$$

Using the equations of this analysis, to solve for $S, p, w$, and $T_{w}$, and arbitrarily defining the weight and wall thickness as unity for a non-segmented radiator with a survival probability of 0.999 , a computer code was developed to tabulate (see Table 1) and plot relative weight $w$ and wall thickness $T_{w}$ for values of $N$ (ranging from 10 to 1000) as a function of $N_{s} / N$, as shown in 
TABLE 1: COMPARISON OF RESULTS FOR OVERALL SYSTEM RELIABILITY S $=0.999$

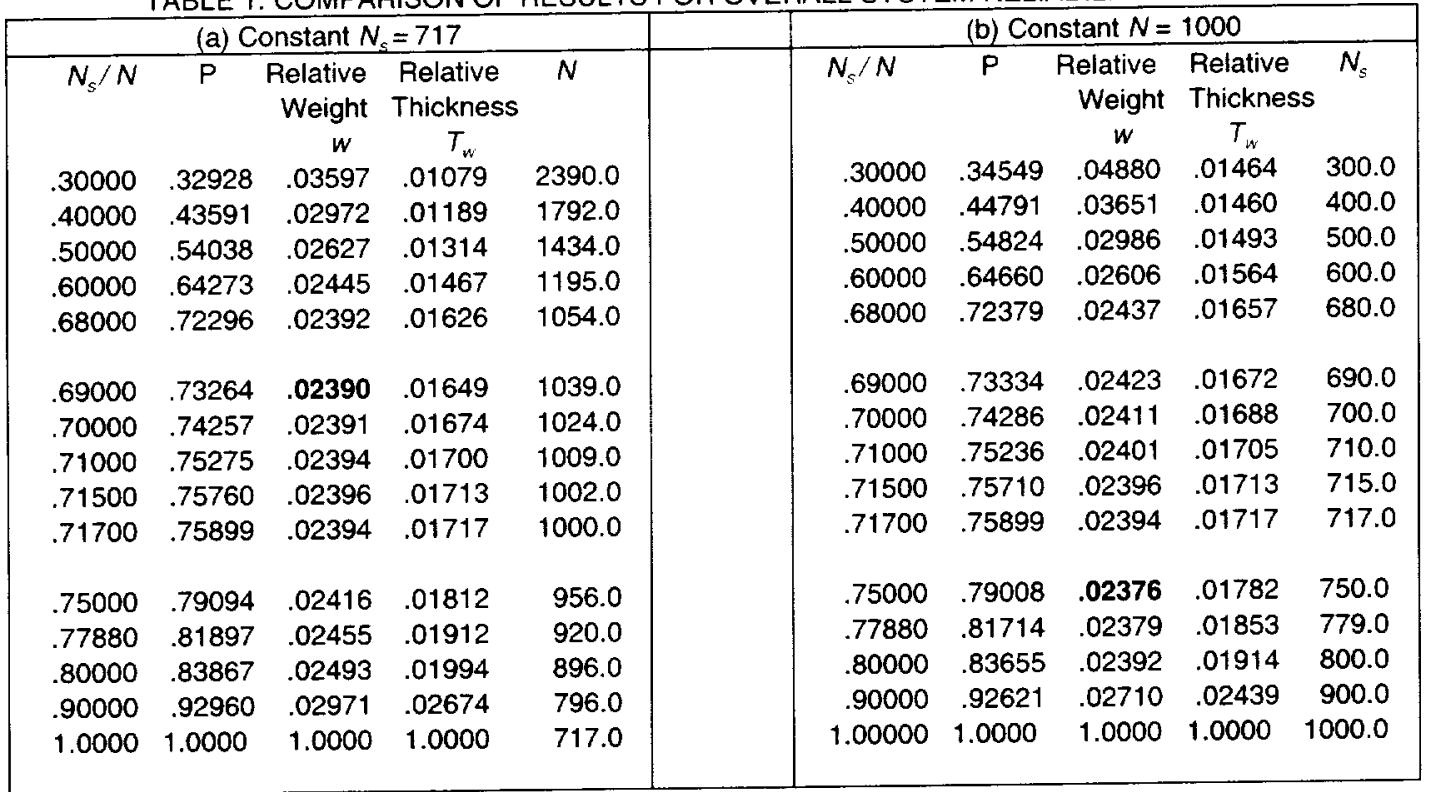
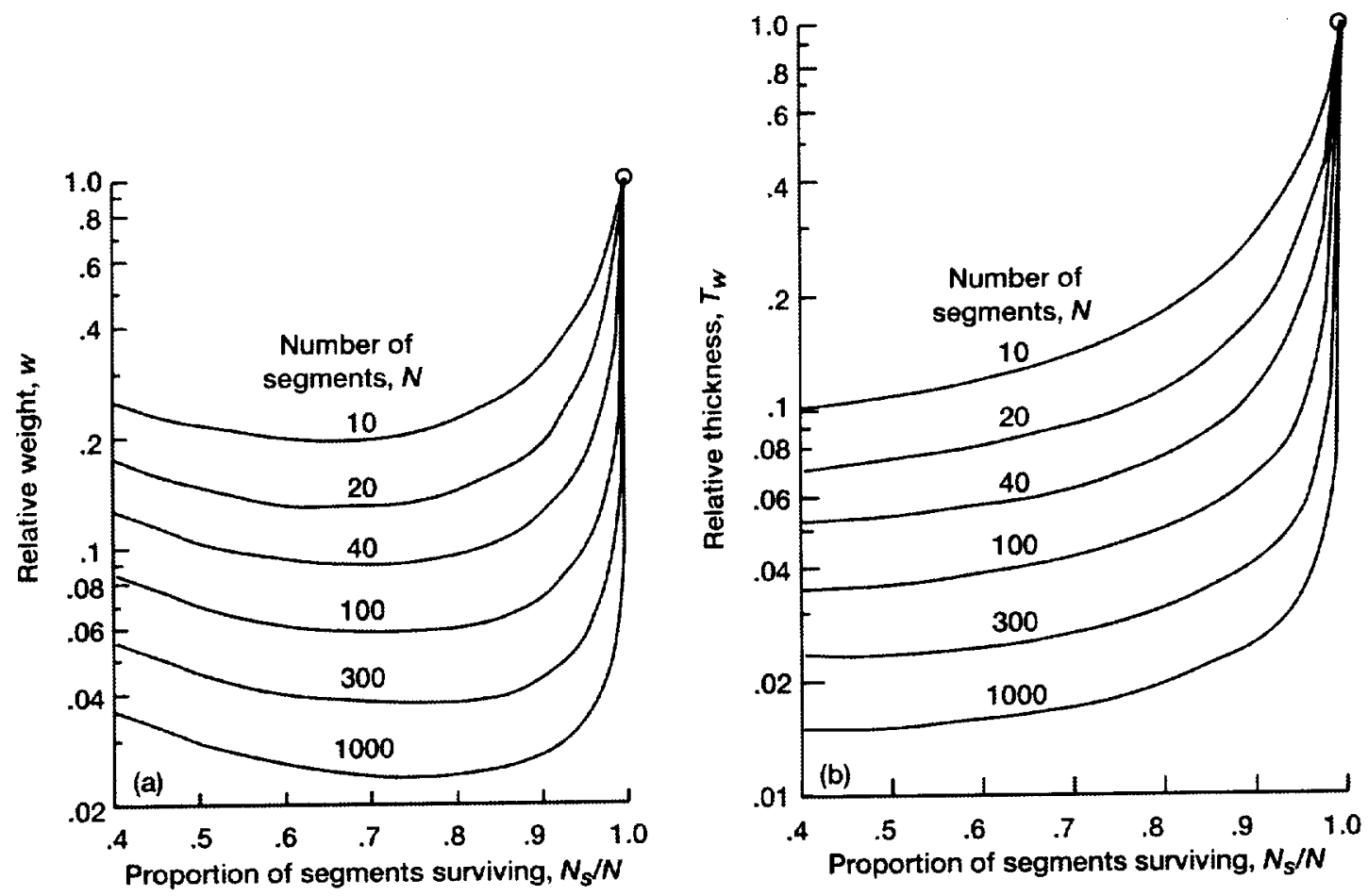

Figure 1.-Segmented heat pipe radiator characteristics for survival probability $S=0.999$. (a) Relative weight of vulnerable surface (nonsegmented $=1$ ). (b) Relative wall thickness (nonsegmented $=1$ ).

figure 1. Note the dramatic reduction in both weight and wall thickness that accrues because of radiator segmenting, even for as few as 10 segments, but especially by increasing the number of segments $N$ to 1000 .

To verify the theoretically derived $N_{s} / N$ ratios at which minimum relative weight is achieved, table 1 shows results for relative weight and thickness for 1,000 segments $(N=1,000)$. Note that the minimum $w$ value (bold number) for constant $N_{s}=717$ occurs at $N_{s} / N \sim 0.69$, rather than the 0.717 value derived in the appendix. Similarly, for constant $N=1000$, the minimum occurs at $N_{s} / N \sim 0.75$ rather than the predicted $e^{-1 / 4}$ value. The reason for this is that the error in the assumption inherent in the derivations, namely $p \sim N / N$, was about 5.5 percent, as can be verified by comparing the tabulated values of $p$ and $N / N$. In table 2 the $p$ values are more in agreement for the higher $N$ values ( $N$ $=3000$ ) and the lower $S$ value (used to reduce the computations). Hence the minimum $w$ values (bold numbers) in table 2 do occur at $N_{s} / N$ values which are in close agreement with the theory. As mentioned above, 
TABLE 2: COMPARISON OF RESULTS FOR OVERALL SYSTEM RELIABILITY $S=0.600$

\begin{tabular}{|c|c|c|c|c|c|c|c|c|c|}
\hline \multicolumn{5}{|c|}{ (a) Constant $N_{s}=2151^{*}$} & \multicolumn{5}{|c|}{ (b) Constant $N=3000^{*}$} \\
\hline$N_{s} / N$ & $P$ & $\begin{array}{c}\text { Relative } \\
\text { Weight } \\
w\end{array}$ & $\begin{array}{c}\text { Relative } \\
\text { Thickness } \\
T_{w}\end{array}$ & $N$ & $N_{s} / N$ & $P$ & $\begin{array}{c}\text { Relative } \\
\text { Weight } \\
W\end{array}$ & $\begin{array}{c}\text { Relative } \\
\text { Thickness } \\
T_{w}\end{array}$ & $N_{s}$ \\
\hline .30000 & .30131 & .02431 & .00729 & 7170.0 & .30000 & .30198 & .03252 & .00976 & 900.0 \\
\hline .40000 & .40164 & .01997 & .00799 & 5377.0 & .40000 & .40211 & .02427 & .00971 & 1200.0 \\
\hline .50000 & .50181 & .01754 & .00877 & 4302.0 & .50000 & .50215 & .01979 & .00989 & 1500.0 \\
\hline .60000 & .60192 & .01619 & .00971 & 3585.0 & .60000 & .60209 & .01718 & .01031 & 1800.0 \\
\hline .68000 & .68197 & .01569 & .01067 & 3163.0 & .68000 & .68197 & .01597 & .01086 & 2040.0 \\
\hline .69000 & .69200 & .01567 & .01081 & 3117.0 & .69000 & .69195 & .01587 & .01095 & 2070.0 \\
\hline .70000 & .70210 & .01565 & .01096 & 3072.0 & .70000 & .70193 & .01577 & .01104 & 2100.0 \\
\hline .71000 & .71203 & .01564 & .01111 & 3029.0 & .71000 & .71191 & .01569 & .01114 & 2130.0 \\
\hline .71500 & .71699 & .01564 & .01118 & 3008.0 & .71500 & .71689 & .01565 & .01119 & 2145.0 \\
\hline .71700 & .71889 & .01564 & .01121 & 3000.0 & .71700 & .71889 & .01564 & .01121 & 2151.0 \\
\hline .75000 & .75184 & .01569 & .01177 & 2868.0 & .75000 & .75181 & .01546 & .01159 & 2250.0 \\
\hline .77880 & .78085 & .01585 & .01234 & 2761.0 & .77880 & .78072 & .01541 & .01200 & 2337.0 \\
\hline .80000 & .80195 & .01603 & .01282 & 2688.0 & .80000 & .80165 & .01544 & .01235 & 2400.0 \\
\hline .90000 & .90128 & .01831 & .01648 & 2390.0 & .90000 & .90117 & .01697 & .01527 & 2700.0 \\
\hline 1.00000 & .99976 & . 12512 & .12512 & 2151.0 & 1.00000 & .99983 & .12512 & .12512 & 3000.0 \\
\hline
\end{tabular}

the relative mass versus $N_{s} / N$ curves are fairly flat near their minimum values, so that the condition shown in (9) will be sufficiently accurate for most applications. The reader may note, that in table 2 the relative mass value $w$ for the nonsegmented radiator $(N / N=1)$ is much lower than unity. This is so because the weight of the nonsegmented radiator at $S=0.6$ is lower than that for a nonsegmented one with $S=0.999$. The relative weight parameter $w$ is referenced to a nonsegmented configuration with a survival probability of 0.999 .

Both, table 1 and figure 1 show that for a given number of segments $N$, the relative weight will increase for values of $N_{s} / N$ below the value given in equation (9), even though the wall thickness decreases. This is because as $N_{s}$ decreases, the vulnerable area of each segment increases and $p$, the probability of no puncture per segment, decreases.

As already mentioned in connection with the derivation of equation (4), fin area, radiator fluid manifolds, and structural weight were not included in this analysis. Regarding fin area, the following observation can be made: If a certain fraction of the vulnerable area $a$ of each segment represents the fin area not rendered useless, even if penetrated by a micrometeoroid, then the results of the present analysis will be conservative (i.e., the relative weights and wall thicknesses will be even lower than shown in fig. 1). However, since the structure and manifolds will need to be protected by a thicker wall or a bumper shield, the benefits provided by fins will be offset to some degree, depending on the particular design of the spacecraft radiator.

Nevertheless, the utilization of finned segments that increase the radiating area without increasing the vulnerable area will provide an additional margin in the design of large segmented space radiators. The use of heat pipes, each operating independently with selfcontained heat transport loops, will further facilitate the space radiator design process.

\section{SEGMENTED RADIATOR DESIGN EXAMPLES}

Having discussed some of the theoretical basis for segmenting radiators and for utilizing heat pipes as ideal elements or building blocks, let us next discuss a few advanced space radiator designs. The conical radiator for the $0.1 \mathrm{MW}$. SP-100 thermoelectric (T/E) space power system with a liquid-metal (lithium) cooled reactor heat source (Juhasz, 1998) is shown in figure 2. The radiator cone is composed of 12 panels, each of which is a radiator segment consisting of $226 \mathrm{C}-\mathrm{C}$ heat pipes of varying condenser length. These heat pipes are essentially of the same design as discussed in this report. The SP-100 radiator, designed for 7-yr survival probability of 0.99 , could provide the $2.4 \mathrm{MW}_{\mathrm{t}}$ heat rejection needs of the power system with only 11 of its 12 panels functioning, each with 75 percent of their original number of heat pipes. Thus each of the twelve panels could absorb the loss of about $\mathbf{5 6}$ heat pipes for a total reduction of radiator area equal to that represented by over 670 heat pipes, without significant impact on the required output power. Note that additional losses in heat pipe radiating surface area would result in a rise in T/E cold junction temperature beyond the design value at the end of life (EOL). As a result the thermal efficiency and thus electric power output would be lowered. It should be noted, however, that no drastic power system failure would occur! Figure 3 shows a proposed heat rejection subsystem for a 15-MW. closed-cycle gas turbine power system with a nuclear high-temperature gas reactor heat 


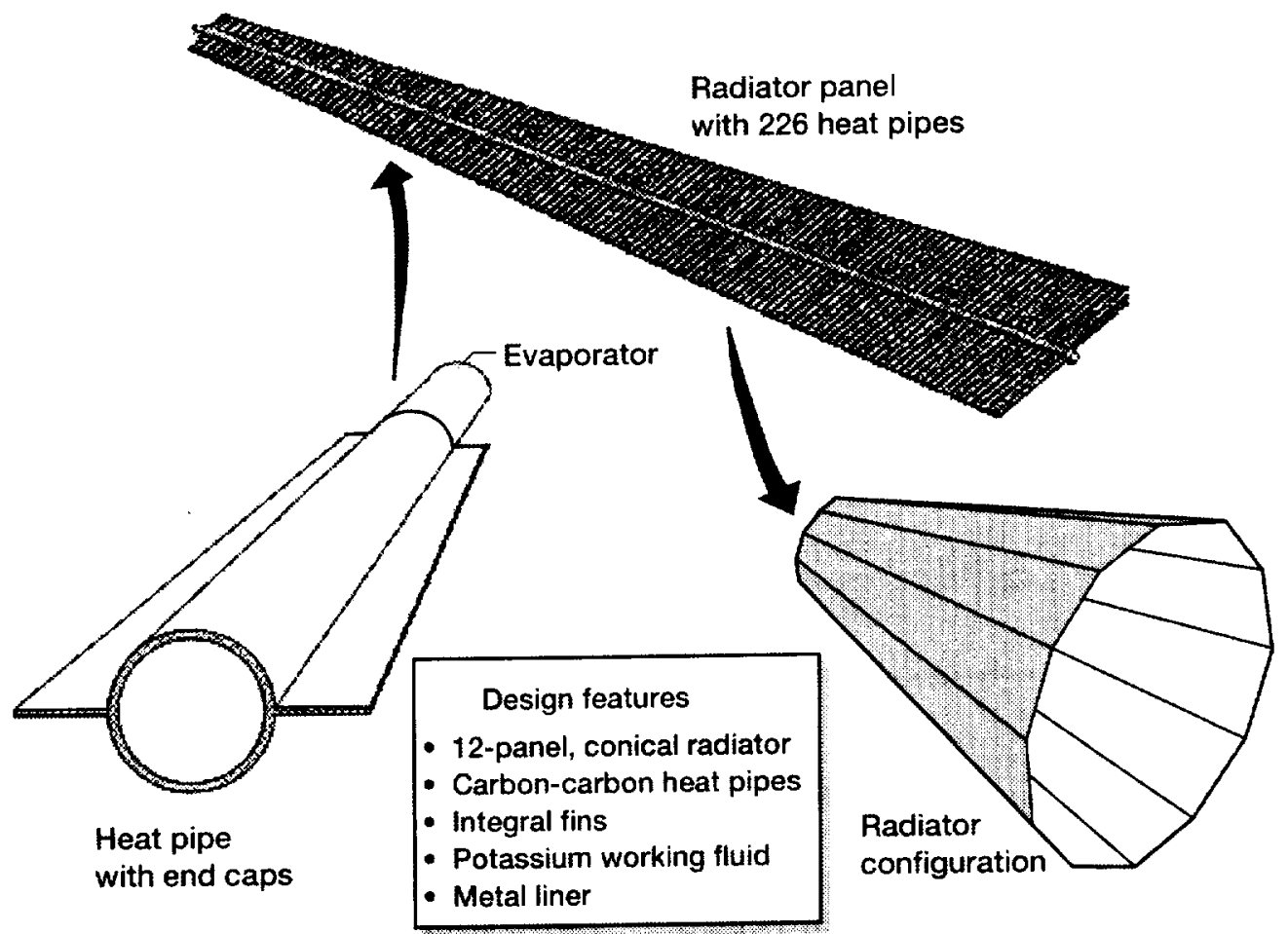

Figure 2.-SP-100 advanced heat pipe segmented radiator.

Inset: Edge view of double $\mathrm{C}-\mathrm{C}$ face sheet configuration
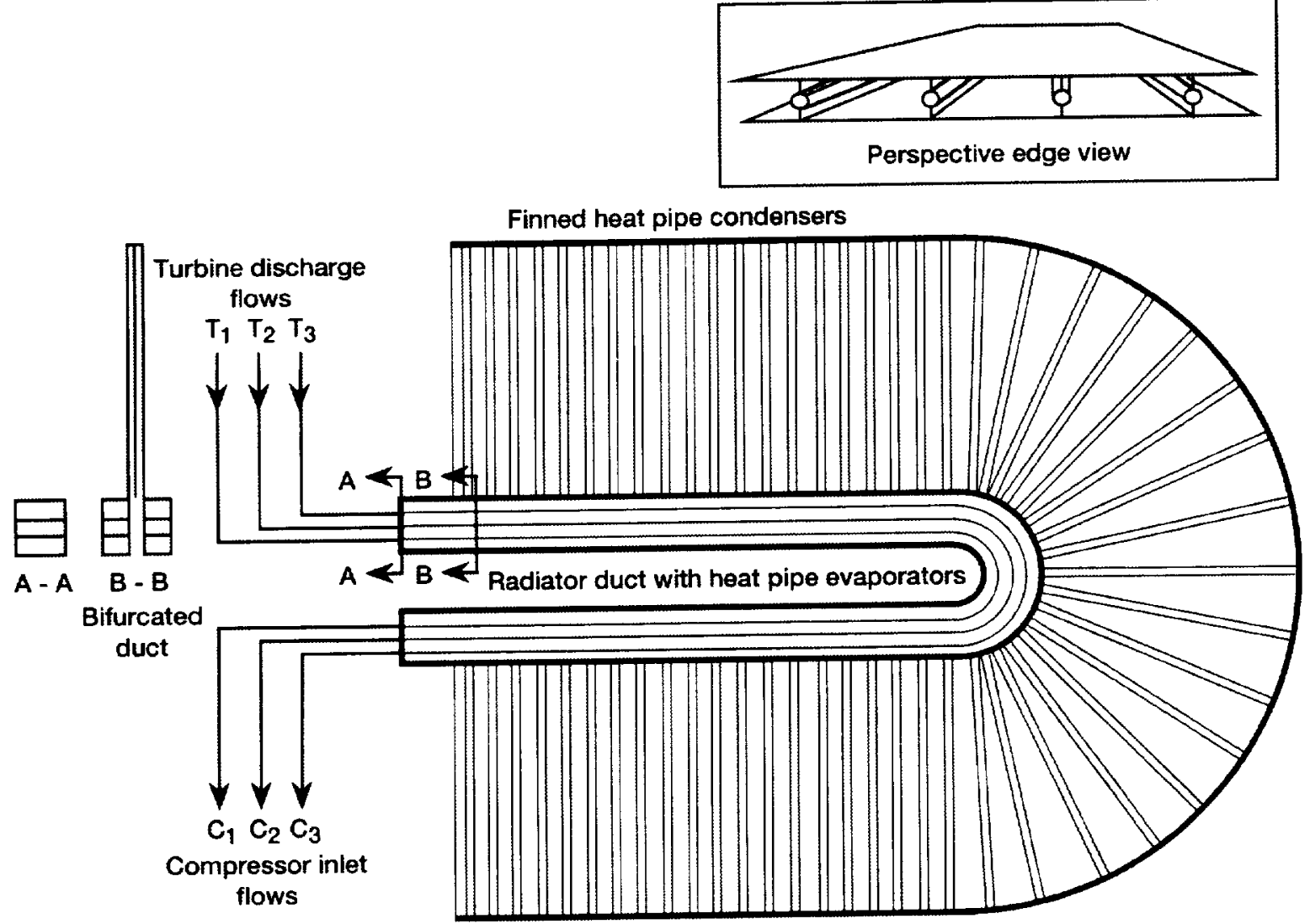

Figure 3.-Flat segmented heat pipe radiator for a nuclear triple loop gas turbine power system. 


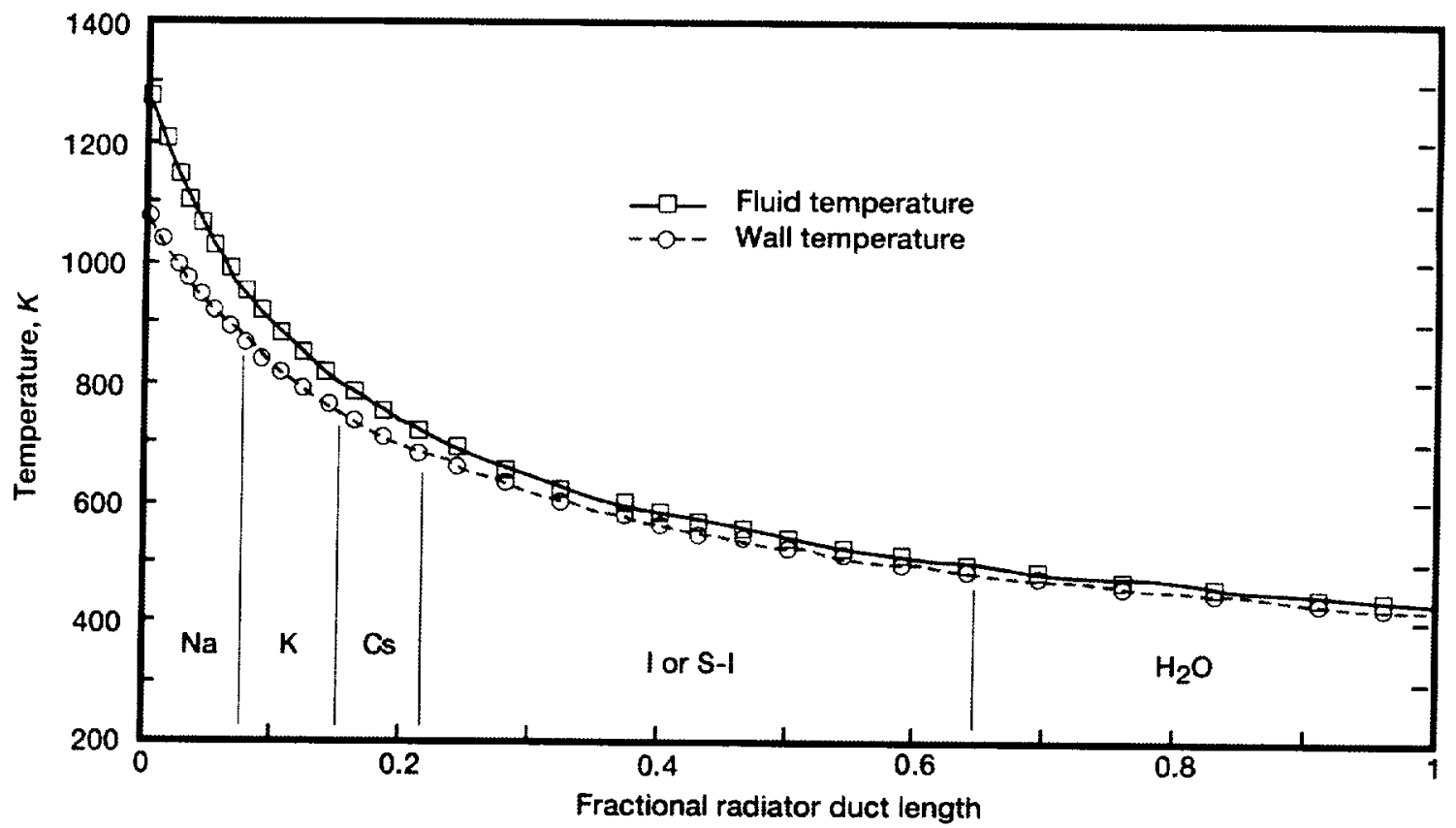

Figure 4.- Heat pipe radiator temperature profiles for a gas turbine power system. (Letters between lines designate heat pipe working fluids.)

source (Juhasz et al., 1993). This segmented radiator system is initially composed of 1600 finned heat pipe segments of which 1200 are needed to survive until the end of the mission. This radiator was designed to meet the heat rejection requirements of three separate gas turbine loops. A bifurcated manifold duct, which carries the He turbine exhaust stream, communicates thermally with $0.05-\mathrm{m}$ diameter, $1.5-\mathrm{m}$ long $\mathrm{C}-\mathrm{C}$ heat pipe evaporators by combined conduction-convection heat transfer. The specific weight of such a radiator was shown to be less than $1.5 \mathrm{~kg} / \mathrm{m}^{2}$ as compared to $\sim 15 \mathrm{~kg} / \mathrm{m}^{2}$ for state-of-the-art (SOA) technology.

The computed temperature profiles for the He gas and the heat pipe evaporator wall along the radiator duct are shown in figure 4 . These profiles were computed under the assumption of constant incremental radiator area with incremental manifold duct length. To accommodate the more than $800-\mathrm{K}$ radiator temperature drop for this high-temperature gas turbine power system (turbine inlet temperature of $2000 \mathrm{~K}$ ), the radiator was divided into five temperature zones, each of which is defined by the operating temperature range associated with a particular heat pipe working fluid: sodium, potassium, cesium, sulfur-iodine (in lieu of mercury), and water. Note that the double $\mathrm{C}-\mathrm{C}$ face sheet configuration suggested by the perspective edge view offers even greater survivability and structural rigidity at a somewhat higher weight, depending on the thickness of the face sheets.

\section{CONCLUDING REMARKS}

A derivation of the mathematical relationships required to evaluate weight and reliability advantage of radiator segmenting was completed, including theoretical analyses of conditions for minimum mass systems. A computer code was also developed to generate analysis results in tabular or graphical form. The drastic reduction in mass and wall thickness required to achieve a required overall reliability for segmented radiators was demonstrated in theory. Several examples of segmented radiator designs utilizing heat pipe elements were described. The advantage of heat pipe technology in fabricating large surface area space radiator systems with multiple redundancy, which achieve high mission reliability at low specific weight, are pointed out in quantitative terms, as compared to non-segmented radiators.

\section{REFERENCES}

1. English, R.E. and Guentert, D.C. (1961): Segmenting Radiators for Meteoroid Protection. ARS Journal, August 1961.

2. Juhasz, A.J. (1998): Design Considerations for Lightweight Space Radiators Based on Fabrication and Test Experience with a Carbon-Carbon Composite Prototype Heat Pipe. NASATP-1998207427, October, 1998.

3. Juhasz, A.J., El-Genk, M.S. (1993), and Harper, W.B.: "Closed Brayton Cycle Power System with a High Temperature Pellet Bed Reactor Heat Source for NEP Applications," Proc. of the $10^{\text {th }}$ Symposium on Space Nuclear Power and Propulsion, AIP 271, Vol. 2, AIP, New York, 1993, pp. 1055-1064. NASA TM-105933.

4. Summers, J.L. and Charters, A.C. (1959): HighSpeed Impact of Metal Projectiles in Targets of Various Materials. Proc. Third Symp. On Hypervelocity Impact (Vol. 1, Armour Res. Foundation of IIT), Chicago, Feb. 1959.

5. Whipple, F.L. (1958): The Meteorite Risk to Space Vehicles. $8^{\text {th }}$ Internat. Astronautical Congress. Springer Verlag 1958, pp. 418-428. 


\section{APPENDIX}

\section{Determination of Minimum Relative Radiator Mass}

The expression for minimum radiator mass relative to an non segmented configuration having a survival probability $S$ of 0.999 was shown in (5) as

$$
w=\left(N / N_{s}\right)\left[N_{s} \ln (1 / p)\right]^{-1 / 3}
$$

It was also stated, that for large values of $\mathrm{N}$ the value of the probability of no puncture of an individual segment, $p$, can be approximated by $N_{s} / N$, i.e., $p \approx N_{s} / N$.

The analysis of two separate cases is of interest.

A. For a given value of surviving segments $N_{s}$ what value of $N_{s} / N$ will minimize $w$ ?

Letting $y=N / N_{s} \approx 1 / p$

The relative mass expression $w$ can be written as

$$
w=y\left[N_{S} \ln y\right]^{-1 / 3}
$$

The minimum of $w$ is found by solving the equation represented by $\partial w / \partial y=0$.

$$
\partial w / \partial y=N_{S}^{-1 / 3}\left\{y\left[-\frac{1}{3}(\ln y)^{-4 / 3} * \frac{1}{y}\right]+1 *[\ln y]^{-1 / 3}\right\}=0
$$

Which can be simplified to

$$
\partial w / \partial y=N_{S}^{1 / 3}(\ln y)^{-4 / 3}\left[-\frac{1}{3}(\ln y)^{-1}+1\right]=0
$$

In (A4) the term in brackets yields:

$$
\text { or } \quad \begin{aligned}
& \ln y=1 / 3 \\
& y=e^{1 / 3}
\end{aligned}
$$

and, since $1 / y=N_{s} / N$, it follows that $N_{s} / N=e^{-1 / 3}$

$$
\text { or }\left(N_{s} / N\right)=0.717
$$

From the above result one may conclude, that with the assumption of a large number of segments, $N$, for which the corollary condition $p \approx N_{s} / N$ also holds, the relative mass $w$ will minimize when the ratio of surviving number of segments to initial number of segments is $\sim 0.72$ if the desired number of surviving segments, $N_{s,}$ is held constant.

\section{Alternate Method:}

The logarithmic form of (A2) is:

$$
\ln w=\ln y-\frac{1}{3} \ln N_{S}-\frac{1}{3} \ln (\ln y)
$$

Differentiating (A2.0) leads to:

$$
\frac{1}{w} \frac{\partial w}{\partial y}=\frac{1}{y}-0-\frac{1}{3} \frac{(1 / y)}{\ln y}=0
$$

Dividing (A2.1) by $1 / y$ it is readily seen that

$$
\begin{aligned}
\ln y & =1 / 3 \\
\text { and } N_{S} / N & =e^{-1 / 3} \equiv 0.717
\end{aligned}
$$

as obtained in (A5.1) above.

B. For a given value of initial segments, $N$, what value of $\mathrm{N}_{s} / \mathrm{N}$ will minimize $\mathrm{w}$ ?

With constant initial number of segments a more convenient form of (A1) is

$$
w=\left(N / N_{S}\right)\left[N\left(N_{S} / N\right) \ln (1 / p)\right]^{-1 / 3}
$$

and with $y=N / N_{s} \approx 1 / p, w$ can be written as

$$
\begin{aligned}
& w=y\left[\frac{N}{y} \ln y\right]^{-1 / 3} \\
= & N^{-1 / 3} y^{4 / 3}(\ln y)^{-1 / 3}
\end{aligned}
$$

Differentiating (B3) and setting the result to zero leads to $\partial w / \partial y=N^{-1 / 3} *$

$$
\left\{y^{4 / 3}\left[-\frac{1}{3}(\ln y)^{-4 / 3} * \frac{1}{y}\right]+\frac{4}{3} y^{1 / 3} *(\ln y)^{-1 / 3}\right\}=0
$$

which can be simplified to

$$
N^{-1 / 3} y^{1 / 3}\left[\cdot \frac{1}{3}(\ln y)^{-4 / 3}+\frac{4}{3} \ln y *(\ln y)^{-4 / 3}\right]=0
$$

and

$$
N^{-1 / 3} y^{1 / 3}(\ln y)^{-4 / 3}\left[-\frac{1}{3}+\frac{4}{3} \ln y\right]=0
$$


In (B6) the term in brackets yields

$$
\ln y=\frac{1}{4} \text { or } y=e^{1 / 4}
$$

and since $\frac{1}{y}=\frac{N_{S}}{N}$, it follows that $\frac{N_{S}}{N}=e^{-1 / 4}=0.7788$

From the result shown in (B8) one may conclude, that with the assumption of a large number of segments, $N$, with the corollary condition $p \approx N_{s} / N$, the relative mass $w$ will minimize when the ratio of surviving number of segments to initial number of segments is $\sim 0.78$ if the desired number of initial segments $\mathbf{N}$ is held constant.
Alternate Method: Writing the logarithmic form of (B3), we have $\ln w=-\frac{1}{3} \ln N+\frac{4}{3} \ln y-\frac{1}{3} \ln (\ln y)$, which can be differentiated to yield

$$
\frac{1}{w} \frac{\partial w}{\partial y}=0+\frac{4}{3} * \frac{1}{y}-\frac{1}{3} * \frac{1}{\ln y} * \frac{1}{y} \equiv \frac{1}{3 y}\left(4-\frac{1}{\ln y}\right)=0
$$

which results in $\ln y=\frac{1}{4} ; y=e^{1 / 4}$ and

$$
\frac{N_{S}}{N}=\frac{1}{y}=e^{-1 / 4} \equiv 0.7788
$$

as obtained in (B8) above. 


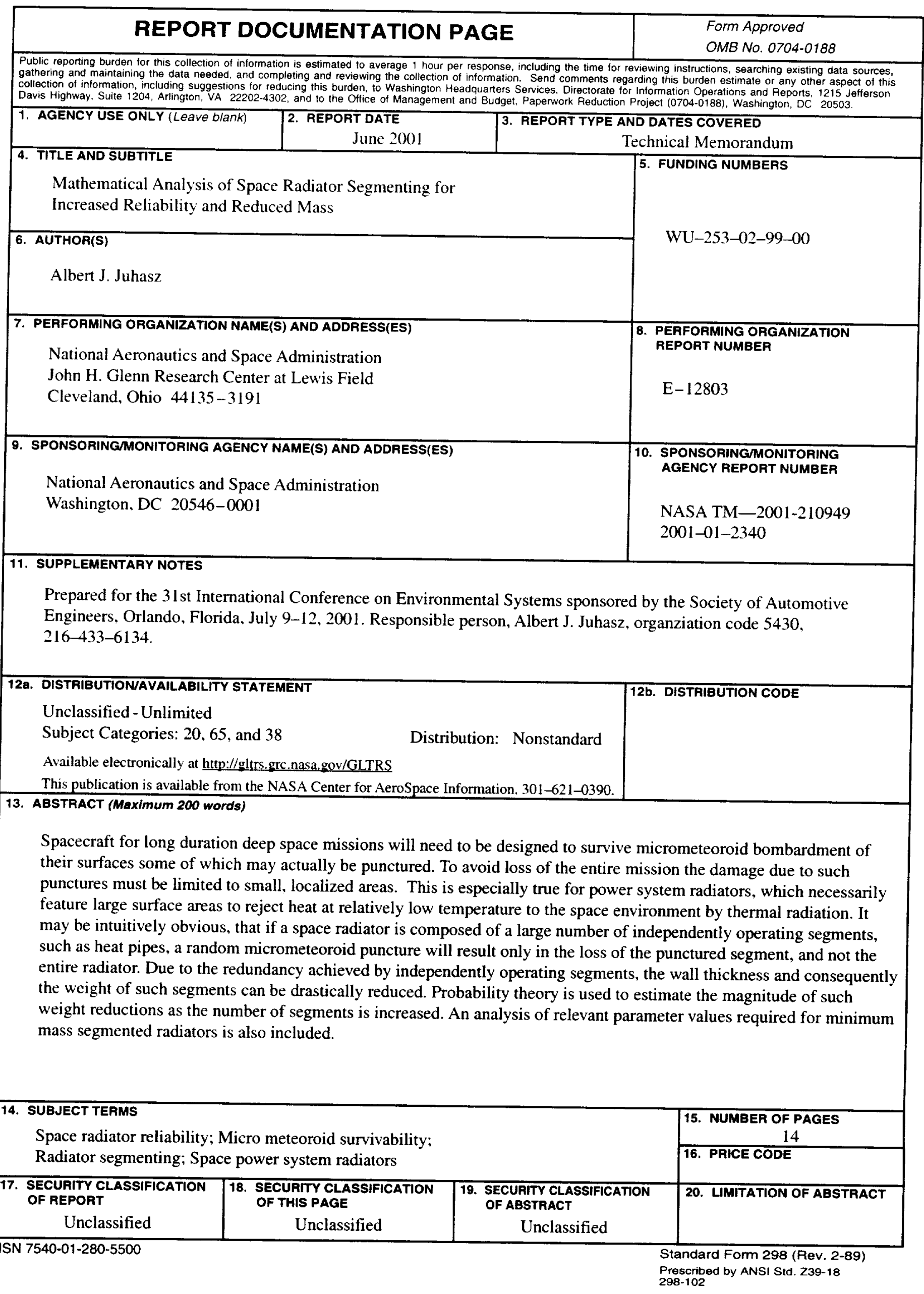

